\title{
Experimental methods for the determination of stress changes at electrified solid-liquid interfaces
}

Gyözö G. Láng*, Noémi Kovács, Soma Vesztergom, Mária Ujvári, Dóra Zalka, Krisztina Szekeres

\section{Eötvös Loránd University, Institute of Chemistry, Department of Physical Chemistry}

H-1117 Budapest, Pázmány P. s. 1/A

\begin{abstract}
In the present study some of the methods developed for the experimental determination of (interfacial or film) stress changes in electrochemical systems containing solid-liquid interfaces are briefly reviewed, as well as the kind and quality of information that can be obtained using these methods are discussed. The "bending beam" ("bending cantilever", "laser beam deflection", "wafer curvature") method and related techniques, the piezoelectric method, the extensometer method, and the method based on the measurement of contact angle are discussed in detail. Special attention has been paid to problems related to the use of optical methods for position sensing.
\end{abstract}

Keywords: solid-liquid interface, solid electrode, electrocapillarity, interface stress

\section{Introduction}

The intensive parameter conjugate to surface area [1] (often called the "surface tension", “interfacial tension", "surface stress", "interface stress" or "specific surface energy") is an important parameter in the thermodynamic theory of electrodes, since most electrochemical systems are, in fact, capillary systems, because any interaction between the bulk solid and the remainder of the system takes place via the interface region. The thermodynamic properties of this region (i.e. the electronic conductor|ionic conductor interface, for instance the interface between a metal and an electrolyte solution) directly influence the electrochemical processes, an understanding of the thermodynamics of interfaces is of importance to all surface scientists and electrochemists. Since the early days of electrochemistry, one of the main goals of research has been to find correlations between the structures formed by surface atoms and adsorbates and the macroscopic kinetic rates of electrochemical reactions.

\footnotetext{
* Corresponding author. Tel.: +36 1209 0555/1107; fax: +36 13722592.

E-mail address: langgyg@chem.elte.hu
} 
It is not surprising, therefore, that the interest in the structure and properties of charged solid |liquid interfaces has increased steadily throughout the past decades and stimulated interesting discussions and debates in the electrochemical community. Nevertheless, the understanding of fundamental aspects of interfacial electrochemistry is also important for other fields, such as materials science, nano-science, biology, medical science, sensor and battery research, as well as for technologies based on electrochemical techniques.

It is known that the formation of an interface between an electronic conductor and an ionic conductor is accompanied by a spatial separation of charge, such that electric potential differences may appear between the adjacent phases. The whole array of charged species, neutral molecules or oriented dipoles existing at a metal|solution interface is called the electrochemical (or electrical) double layer.

Electrocapillarity is the study of the interfacial tension (stress) as a function of the electrode potential (the term "electrode" is used here to denote heterogeneous electrochemical systems, in which at least two phases are connected and one of them is an electronic conductor or a semiconductor, the other is an ionic conductor, usually an electrolyte solution). Precise quantitative measurements of interfacial tensions have been obtained for liquid | liquid interfaces as reported, for example, refs. [2-7]. The interpretation of electrocapillary data obtained with liquid metals in contact with electrolyte solutions is greatly facilitated by the concept of ideal polarizability. For perfectly (ideally) polarizable electrodes the relationship between the interfacial tension, the electrode potential and the surface charge density can be described by the Lippmann equation [8].

On the other hand, a study of the interface stress of a "solid electrode" (i.e. an electrode that contains an electronically conducting solid phase in contact with an ionically conducting liquid phase) is complicated by many factors, and with the exception of a few special situations the absolute interfacial tension at the solid|liquid interface is inaccessible by experimental methods. In addition, a solid does not generally exhibit an "equilibrium" surface. According to [9]: "their surface will be largely a frozen-in record of an arbitrary past history". Although a change in a thermodynamic parameter may not result in full equilibrium, the system can escape from the constraint by optimization of, for example, "the distribution of imperfections, surface waves and humps" [10] in the rational time scale, or more generally, it will approach relative minima of the Gibbs energy of the interface. Nevertheless, during the past decades several attempts have been made to derive thermodynamic equations for the solid|liquid interface, and several methods were suggested (and were supposed to be suitable) for the determination of 
changes in the interface stress ("specific surface energy", "interfacial tension", "surface stress") of solid electrodes.

As a general rule, experimental data should be compared to theoretical predictions and calculations. Unfortunately, for solid electrodes the interpretation of the results from various methods in terms of physicochemical properties of the system is not without problems [11-35].

In principle, the quantitative predictions made by the theory can be tested experimentally. Unfortunately, interface stress changes of solid/liquid interfaces are very difficult to measure owing to the lack of reliable methods. Theoretical estimates of absolute surface tension of some relatively simple covalently bonded, ionic, rare-gas, and metallic crystals are discussed in the literature [36]. In a few specific situations, the surface tensions of some solid surfaces have been determined experimentally. These experimental methods are designed for the solid/gas interface, and are mostly incompatible for use at room temperature or in the presence of an electrolyte solution. Consequently, they cannot be applied to study the surface energetics of solid electrodes. On the other hand, during the past decades several attempts have been made to derive thermodynamic equations for the solid/liquid interface, and several methods were suggested for measurements of changes of the interface stress of solid electrodes [37-48].

According to the classification proposed by Morcos [49] attempts to determine the interface stress change of solid electrodes fall into two main categories: measurement of the potential dependence of contact angle established by liquid phase on the solid surface [34,50-54] and the measurement of the variation in interface stress experienced by the solid as a function of potential. (In the present study, we focus on the latter case.)

Variation in the interfacial stress may either be measured "directly" [55,56], with a piezoelectric element, or be obtained indirectly [46,57-60], by measuring the potential dependence of the strain (i.e. electrode deformation) and then obtaining the variation in stress from the appropriate form of Hooke's law. We would like to stress here again that the above methods only yield changes of surface stress as a function of various physicochemical parameters e.g. as a function of electrode potential, and in principle, if there are both "plastic" and "elastic" contributions to the total strain, the changes of the "generalized surface parameter" [1] can be determined.

Unfortunately, most of the proposed methods have drawbacks; i.e., they are technically demanding, they cannot be used to monitor changes of the surface stress, they are semiempirical and depend on unverified assumptions, etc. 
In the present work, a brief review is given of some techniques proposed for the determination of changes of interface stress of "solid electrodes" ("bending beam", "bending cantilever", "laser beam deflection", "wafer curvature" or "bending plate/disc" methods [e.g. 34,37, 40,61], laser interferometry [e.g. 34,37,57,60,62-64], piezoelectric method [e.g. 65-67], extensometer method and its variants [e.g. 68,69]) as well as the kind and quality of information that can be achieved using these methods are discussed.

It should be noted that in most of the literature reviewed here the intensive parameter conjugate to the surface area is usually called "surface tension" or "surface stress" and is denoted generally by $\gamma_{\mathrm{s}}$ or $\sigma_{\mathrm{s}}$, respectively. In order to be consistent with the original literature, wherever and whenever possible, we will keep the "original" notation throughout the paper.

\section{Experimental methods}

\subsection{The extensometer method and related techniques}

Beck et al. [68-71] attempted to determine variations in surface stress as a function of potential by using an extensometer which measures the corresponding variation in the length of a very thin metal ribbon. (The results published more recently in ref. [72] are also noteworthy.) In the extensometer device the ribbon in contact with an electrolyte solution served as a working electrode in an electrochemical cell. The ribbon was kept under an approximately constant force (a mechanically applied tensile stress) throughout the experiment by mounting it axially inside a glass tube. As depicted in Fig. 1, the upper end of the ribbon or wire is attached to a spring. The spring constant of the spring should be small compared with the stiffness of the ribbon. The lower end of the ribbon is attached to the glass tube through a Teflon plug. An aluminum bobbin mounted on the quartz spindle forms two capacitors with fixed plates mounted in the head. Small changes in the two capacitances due to length changes of the ribbon are measured with an electronic capacitance sensor. The output voltage $V(\Delta L)$ and the change in the length of the ribbon is described by the relationship:

$$
V(\Delta L)=K_{1}\left[\left(1 / C_{1}\right)-\left(1 / C_{2}\right)\right]=K_{2} \Delta L,
$$

where $C_{1}$ and $C_{2}$ are the two capacitances and $K_{1}$ and $K_{2}$ are proportionality constants.

The variation in surface stress, $\Delta \gamma_{\mathrm{s}}$, can be obtained from the change in the ribbon length $\Delta L$ by an equation developed by Beck: 


$$
\Delta \gamma_{\mathrm{s}}=-\frac{A E}{P L} \Delta L
$$

where $A$ and $P$ are the cross-sectional area and periphery of the ribbon and $E$ is Young's modulus (Fig. 1). We refer the reader to [34] and references therein for more details on the derivation of Eqs. 1 and 2.

Unfortunately, thermal expansion constitutes a serious problem in the extensometer method. The error due to thermal expansion can be reduced, but unless the effect on thermal expansion can be quantitatively accounted for, the results of the extensometer method cannot be conclusively interpreted.

In [73] the effect of mechanical stress on electrode potential $E$ was studied under zero current conditions. The experimental setup was very similar to the extensometer described above. A tensile stress machine was used for mechanical characterization of the samples. A two-electrode geometry was used for the potential measurements, the potential of a thin Ag wire made taut vertically in an electrolyte solution $\left(\mathrm{AgNO}_{3}\right)$ was measured against a reference electrode with a high input impedance voltmeter. The total length of the wire was $50 \mathrm{~mm}$, the portion in contact with the electrolyte solution was $30 \mathrm{~mm}$. One end of the wire was fixed to one arm of a balance the other arm of which was loaded with standard weights. The applied force was low enough to maintain proportionality between stress and strain (elastic deformation conditions).

The numerically large response reported in [73] has been questioned in [74]. It has been pointed out, that the $\mathrm{d} E / \mathrm{d} \sigma$ values (where $\sigma$ is a measure for tangential stress, which scales with the elastic strain $\varepsilon$ ) reported in the literature $[75,76]$ differ by several orders of magnitude and even by sign. In [74] the measurement of the response of the electrode potential $(\Delta E)$ of a polarizable electrode (gold $\mid 10 \mathrm{mmol} \cdot \mathrm{dm}^{-3}$ aqueous perchloric acid solution) to elastic strain under open circuit has been described. A lock-in technique was used to measure the potential variation during cyclic elastic deformation of a thin film electrode supported on a polymer substrate. The method allowed the potential-strain response to be accurately resolved for elastic strain amplitudes as small as $10^{-4}$. It has been shown that the approach similar to that originally suggested by Gokhshtein (see the piezoelectric method discussed below) can provide quantitative data for $\mathrm{d} E / \mathrm{d} \varepsilon$. Cyclic voltammetric experiments combined with dilatometric detection of the length change have been reported in $[77,78]$.

In refs. $[79,80]$ a laser technique, based on optical fiber interferometry, is described for in situ measurements of electrode strain during electrode reactions. The basic concept utilizes a metal 
coated optical fiber in contact with an electrolyte solution as the working electrode in an electrochemical cell, while simultaneously using the fiber as one arm of a Mach-Zehnder interferometer [34].

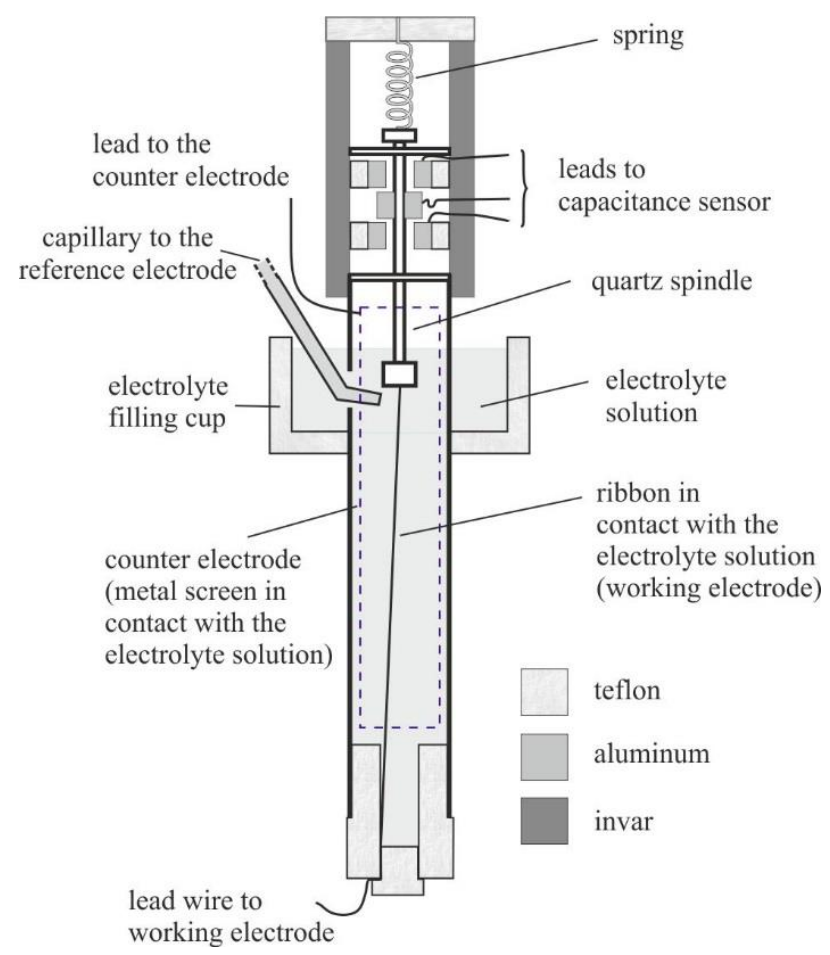

Figure 1: Schematic design of the extensometer

\subsection{Piezoelectric method}

According to our knowledge, Gokhshtein $[55,81,82]$ was the first to measure changes $\partial \gamma_{\mathrm{s}} / \partial E$ of the interface stress ("surface tension", "interfacial tension") $\gamma_{\mathrm{s}}$ with the electrode potential $E$ at platinum electrodes in sulfuric acid using the "piezoelectric" method. The method further improved by various authors $[56,65,83,84]$, especially by Seo et. al. $[56,66,67]$ is "direct" in the sense that it is the variation in the electrode deformation that is "registered" directly by a piezoelectric element. A metal plate is rigidly connected, in a special manner, to a highly sensitive piezoelectric element (Fig. 2).

The applied potential consists of a mean (DC) component upon which is superimposed a high-frequency (AC) component (usually a sinusoidal signal is superimposed on a linear potential sweep). Electrode potential oscillation with the amplitude $\Delta E$ result in oscillation with an amplitude $\Delta \gamma_{\mathrm{s}}$ in the interface stress, which in turn set up forces of inertia that excite vibrations in the entire electrode-piezoelement unit. 
The piezoelectric element converts the mechanical oscillations to alternating electric signals, which can be detected by using a lock-in amplifier, an oscilloscope or a frequency response analyzer. In fact, this is some kind of electromechanical immittance measurement. The geometry of the electrode and its oscillation is of no fundamental importance. The metal in contact with the electrolyte solution may be made in the form of a tight filament which can make lengthwise oscillations, a straight rectangular rod which can make bending oscillations, etc. The alternating surface stress sets in motion not only the electrode, but the whole electrode and piezoelement system, since the inertia forces are essential.

The oscillations of surface stress can also be excited by the application of high-frequency current. In this case the amplitude of the surface charge density $(q)$ is kept constant by specifying the amplitude of the alternating current. According to [55] under such conditions $\Delta \gamma_{\mathrm{s}}$ will be proportional to the derivative $\partial \gamma_{\mathrm{s}} / \partial E$, which is called the " $q$-stance". If the electric variable is the potential, and the measurement is carried out with a constant amplitude $\Delta E$, the amplitude of the piezoelectric voltage $|A|$ is proportional to the derivative of the surface stress with respect to the electrode potential $\left|\partial \gamma_{\mathrm{s}} / \partial E\right|$, and the phase angle $(\phi)$ contains information about the change in the sign of $\partial \gamma_{s} / \partial E$. The $\partial \gamma_{s} / \partial q$ value is related to $\partial \gamma_{s} / \partial E$ (designated by Gokhshtein as $\varepsilon$-stance and $\varphi$-stance, respectively [55]) by

$$
\frac{\partial \gamma_{\mathrm{s}}}{\partial q}=\frac{\partial \gamma_{\mathrm{s}}}{\partial E} \frac{\partial E}{\partial q}=\frac{1}{C} \frac{\partial \gamma_{\mathrm{s}}}{\partial E}
$$

where $C$ is the electrode capacitance.

By applying the piezoelectric method $\partial \gamma_{\mathrm{s}} / \partial E$ is measured at high frequencies and the quantitative determination of interface stress changes requires a sophisticated calibration procedure (the transfer function of the mechanical coupling is rather complicated). However, the potentials of extrema of the function interface stress vs. potential can be obtained directly. The relation between the piezoelectric signals $(|A|$ and $\phi)$ and the $\Delta \gamma_{\mathrm{s}}$ vs. $E$ curve is shown schematically in Fig. 3.

A series of measurements has been performed to date in order to understand electrode processes such as electrosorption and initial oxidation. This technique was capable of detecting sensitively the shift in potential of zero charge (pzc) due to the adsorption of ions and the sign reversal of surface charge due to the formation and reduction of surface oxide phases. E.g. in case of platinum is sulfuric acid solutions Gokhshtein observed two extrema in the hydrogen adsorption region [46]. Similar results were obtained by Seo et al. [56] applying the same experimental method to platinum in $0.5 \mathrm{M}$ acid sulfate solutions. On the other hand, Malpas et al. 
[65] observed only one extremum at $E \approx 0.05 \mathrm{~V}$ for platinum in $0.1 \mathrm{M}$ sulfuric acid. The electrode potential of the maximum was found to shift with $\mathrm{pH}$ to more negative values according to $\partial E_{\mathrm{m}} / \partial \mathrm{pH}=-40 \mathrm{mV}[56]$.

Obviously, because of the dynamic features of the method, the recorded variation in surface stress does not always correspond to equilibrium conditions. For nearly ideally polarizable electrodes, e.g. Au in contact with aqueous sodium-sulfate solution in a certain electrode potential range, equilibrium may be reached during the measurement, because the time for charging/discharging of the electrochemical double layer is shorter than the period of oscillation of the interfacial tension. In case of platinum the period of oscillation is shorter than the time necessary for adsorption of hydrogen or oxygen to reach equilibrium, thus the results depend on the frequency of oscillation as well as on the rate on the scan rate of the linear sweep.

On the other hand, an important advantage of the piezoelectric method is the selective separation of surface energy contributions from other side effects, such as changes in bulk stress due to diffusion or Joule heating of the electrolyte solution [55].
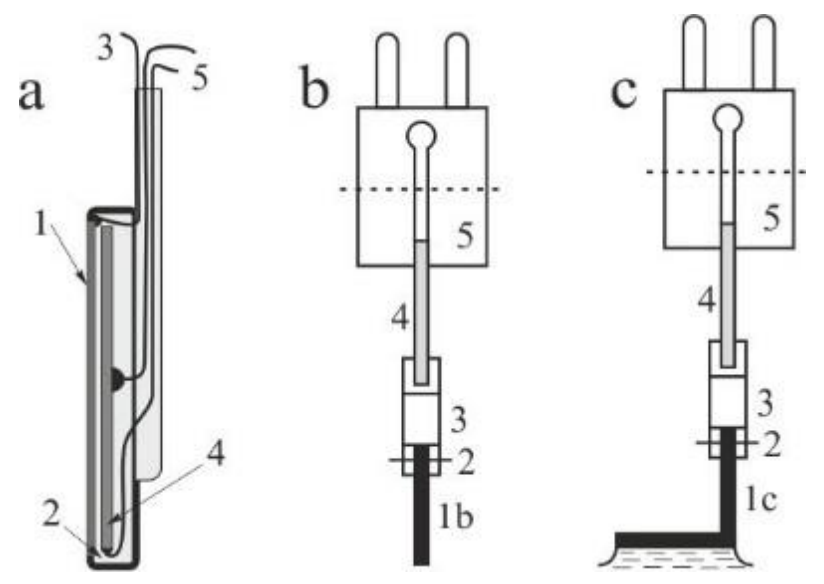

Figure 2: Schematic illustration of devices for the "piezoelectric method". a: A metal plate is rigidly connected to a highly sensitive piezoelectric element; 1 : metal foil; 2: epoxy cement; 3: lead to potentiostat; 4: piezoelectric ceramic disk or plate; 5: leads to amplifier or analyzer; b,c: Piezoelement units designed by Gokhshtein. $\mathrm{b}$ : with plunger, c: with foot. 1a, 1b: metal plate, rod or filament; 2: holder; 3: rib; 4: piezoelement; 5: plug; 


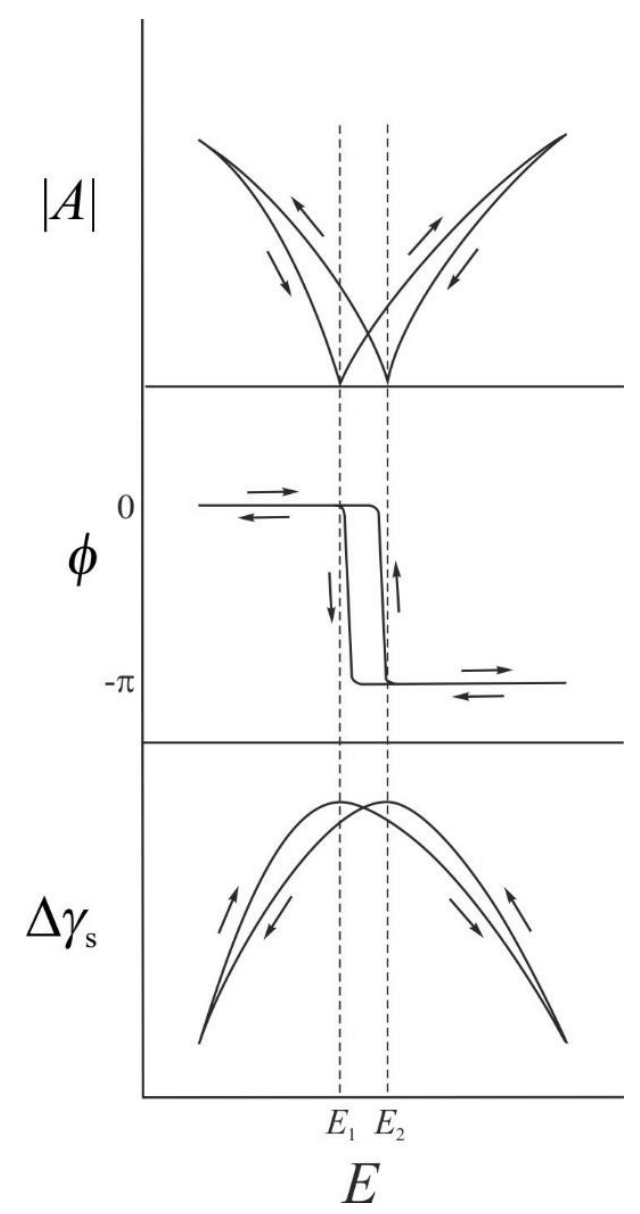

Figure 3: Measured quantities in a typical piezoelectric experiment: the amplitude of the piezoelectrical signal $|A|$, the phase angle $\phi$, and the change of surface stress $\Delta \gamma_{\mathrm{s}}$, as a function of the electrode potential $E$. Numerical integration of the experimentally determined $|A|$ curve with respect to the electrode potential, yields a quantity proportional to $\Delta \gamma_{\mathrm{s}}$.

\subsection{Bending beam and bending plate methods}

The principles of the "bending beam" ("bending cantilever", "laser beam deflection", "wafer curvature", etc.) method were first stated by Stoney $[85,86]$, who exploited the stressinduced curvature of a thin plate (steel rule), which served as a substrate to investigate the stress of an electrodeposited $\mathrm{Ni}$ film. He derived an equation relating the film stress $\sigma$, film thickness $t_{\mathrm{f}}$ to the radius of curvature $(R)$ of the beam 


$$
\sigma t_{\mathrm{f}}=\frac{E_{\mathrm{S}} t_{\mathrm{S}}^{2}}{6 R}
$$

where $t_{\mathrm{S}}$ is the substrate thickness, and $E_{\mathrm{S}}$ is the modulus of elasticity (Young's modulus) of the substrate. This equation is sometimes rewritten by introducing the "isotropic surface stress" $\left(\gamma_{\mathrm{s}}\right)$ as

$$
\gamma_{\mathrm{s}}=\frac{E_{\mathrm{S}} t_{\mathrm{S}}^{2}}{6 R}
$$

Since the publication of the classical paper of Stoney [85] both equations (4) and (5) have been referred to as Stoney's formula or Stoney's equation.

It should be noted here, that original Stoney equation given above is not valid in general, as it describes a one-dimensional stress-curvature scenario. This is not fulfilled in most applications. In order to account for the biaxial nature of the stress we have to replace $E_{\mathrm{S}}$ by $E_{\mathrm{S}} /\left(1-v_{\mathrm{S}}\right)$, where $v_{\mathrm{S}}$ is the substrate Poisson's ratio $[34,87,88]$, and the film stress can be given by

$$
\sigma=\frac{E_{\mathrm{S}} t_{\mathrm{S}}^{2}}{6 t_{\mathrm{f}}\left(1-v_{\mathrm{S}}\right) R},
$$

and

$$
\gamma_{\mathrm{s}}=\frac{E_{\mathrm{S}} t_{\mathrm{S}}^{2}}{6\left(1-v_{\mathrm{S}}\right) R} .
$$

The above equations can be valid under the following conditions: (a) The substrate is homogeneous, linearly elastic, and uniformly thick. (b) The stress is uniform throughout the film thickness. (c) The substrate thickness is much greater than the film thickness. (According to [89]) the $t_{\mathrm{f}} / t_{\mathrm{s}}$ ratio should be $\leq 10^{-3}$.) (d) the radius of curvature of the substrate is much greater than the thickness of the composite structure, i.e. the bending displacement is small compared to the thickness of the substrate. We refer the reader to [34] and references therein for more details on the derivation of Eqs. 6 and 7 .

Measuring the bending of a plate or strip to determine interface stress change or the stress in thin films is a frequently used technique in electrochemistry [37-48]. The electrochemical application of the "bending beam" technique is based on the fact that the changes of the surface stress $\left(\Delta \gamma_{\mathrm{s}}\right)$ for a thin metal film on one side of an insulator strip (or a metal plate, one side of which is coated with an insulator layer) in contact with an electrolyte solution can be estimated from the changes of the radius of curvature of the strip. Usually, in such experiments 
a thin metal strip, or a thin strip of glass or other substrate (on which the metal film is deposited) is rigidly clamped at one end in a fixed mount to form a cantilever. The deflection of the free end, as the strip becomes bent, is then measured by some means. The method has been applied for instance for the investigation of the origin of electrochemical oscillations at silicon electrodes [90] or in the course of galvanostatic oxidation of organic compounds on platinum $[60,61]$, for the study of volume changes in polymers during redox processes [91], for the investigation of the response kinetics of the bending of polyelectrolyte membrane platinum composites by electric stimuli [92], and for the experimental verification of the adequacy of the "brush model" of polymer modified electrodes [93], for in situ monitoring of the electrochemical degradation of polymer films on metals [94-97]. In refs. [98] and [99] the reduction of perchlorate ions at ruthenium electrodes was investigated by voltammetry, chronoamperometry, impedance spectroscopy, and by measuring changes of interfacial stress changes using the cantilever bending method as functions of electrode potential, and concentrations of perchloric acid and $\mathrm{HCl}$. The results are consistent with the earlier observation that perchlorate ions can be electrochemically reduced to $\mathrm{Cl}^{-}$on ruthenium.

As it has already been mentioned above, the "bending beam" method can be effectively used in electrochemical experiments. If the potential of the electrode changes, electrochemical processes resulting in the change of $\gamma_{\mathrm{s}}$ can take place exclusively on the metal side of the sample. The change in the surface stress induces a bending moment and the strip bends. In case of a thin metal film on a substrate if the thickness of the film $t_{\mathrm{f}}$ is sufficiently smaller than the thickness at of the plate, $t_{\mathrm{S}}>t_{\mathrm{f}}$, the change of $\gamma_{\mathrm{s}}$ can be obtained by an expression based on a generalized form of Stoney's equation

$$
\Delta \gamma_{\mathrm{s}}=k_{\mathrm{i}} \Delta(1 / R)
$$

where $k_{\mathrm{i}}$ depends on the design of the electrode. In the simplest case it can be given as (see Eq.7):

$$
k_{\mathrm{i}}=\frac{E_{\mathrm{s}} t_{\mathrm{s}}^{2}}{6\left(1-v_{\mathrm{s}}\right)}
$$

where $E_{\mathrm{S}}, v_{\mathrm{S}}$, and $R$ are Young's modulus, Poisson's ratio and radius of curvature of the plate, respectively. The derivation of Eq. 8 implies the assumption that $\Delta \gamma_{\mathrm{s}}=t_{\mathrm{f}} \Delta \sigma$, where $\Delta \sigma$ is the change of the film stress. It should be noted that a number of authors have tried to modify Stoney's approach over the years, and several modified equations have been derived, however, these equations can usually be written in a form equivalent to Eq. 8 [100-104], according to 
which the change of the reciprocal radius $\Delta(1 / R)$ of curvature of the plate (i.e. the change in the curvature) has to be known for the determination of $\Delta \gamma_{\mathrm{s}}$.

The values of $\Delta(1 / R)=\Delta \gamma_{\mathrm{s}} / k_{\mathrm{i}}$ can be calculated,

(a) if the changes of the deflection angle of a laser beam mirrored by the cantilever are measured using an appropriate experimental setup (Fig. 4), or

(b) the deflection of the plate is determined directly, e.g. with a nanointender, an atomic force microscope (AFM), or a scanning tunneling microscope, etc.

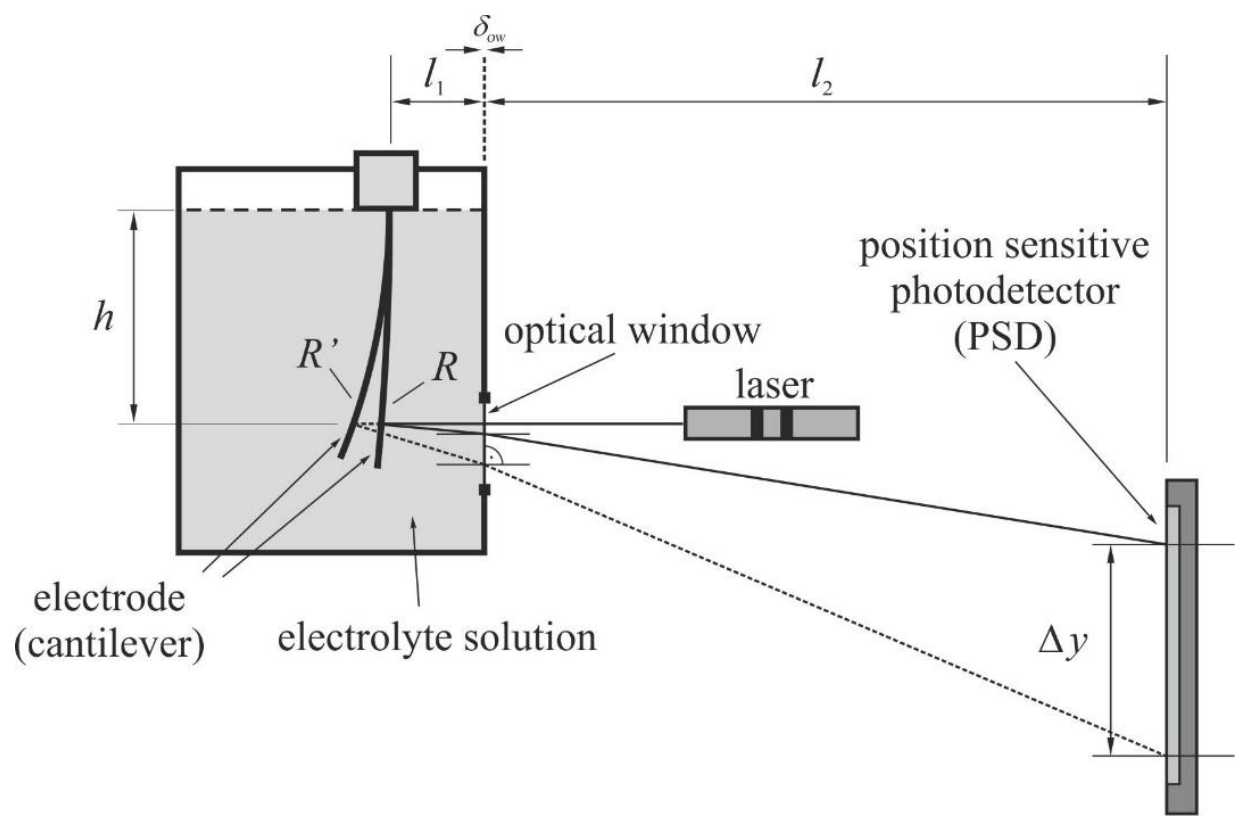

Figure 4: Schematic representation of a typical electrochemical (optical) bending beam setup (normal incidence of the light beam). $\Delta y$ is the displacement of the light spot on the position sensitive detector (PSD) if the radius of curvature changes from $R$ to $R^{\prime}, l_{1}$ is the distance between the clamped end of the probe (cantilever, electrode) and the optical window, $l_{2}$ is the distance between the optical window and the photodetector (PSD), $h$ is the distance between the solution level and the reflection point, $\delta_{\text {ow }}$ is the thickness of the optical window, respectively.

\subsubsection{Optical detection}

\subsubsection{Direct position sensing}

Fig. 5 shows a possible arrangement for electrochemical bending beam experiments with optical detection $[34,105]$. Such a setup can be used mainly for the investigation of small 
deflections, and several details may be different in special cases. E.g. a multi-beam optical sensor (MOS) technique was used by Proost et. al. in [106,107]. With this technique, the spacings between a one-dimensional array of multiple laser reflections of the cantilevered substrate can be continuously monitored with a charge coupled device (CCD) camera (Fig. 6). The multibeam technique has been successfully used for in situ monitoring of the stresses generated in metal or ceramic thin films deposited in vacuum by chemical vapor deposition, physical vapor deposition or molecular beam epitaxy [108-115].

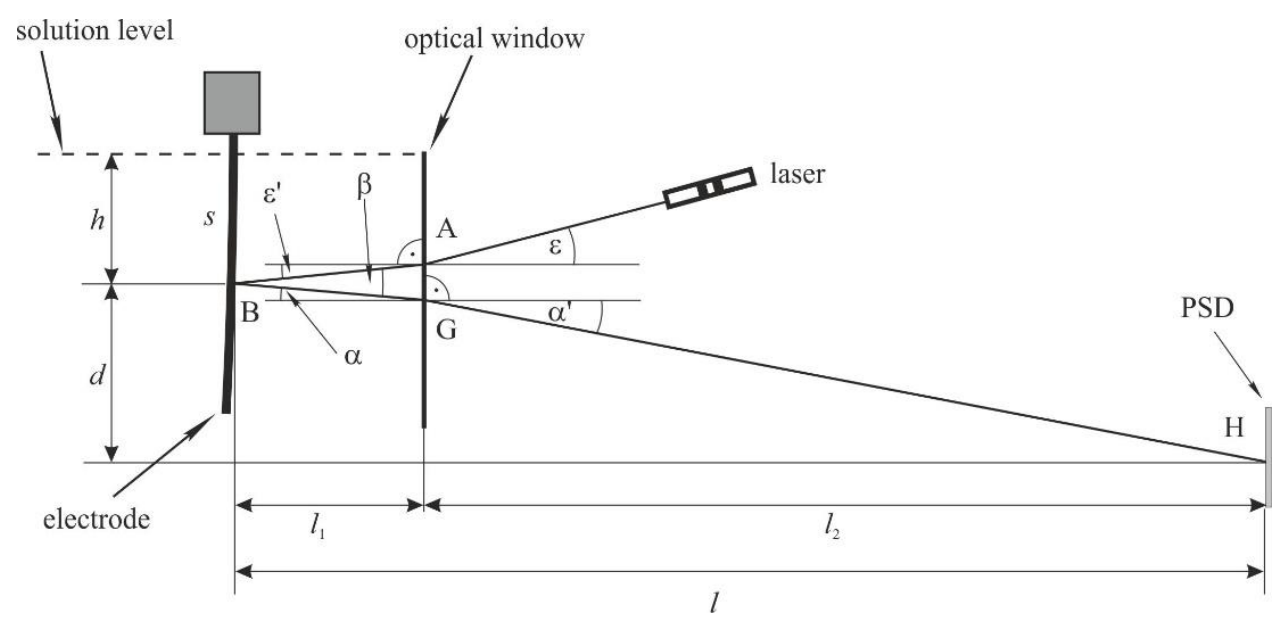

Figure 5: Optical configuration of the bending beam experimental setup in a system consisting a liquid phase (non-normal incidence of the light beam). $\varepsilon$ : the angle of incidence of the light beam coming directly from the laser (in air), $\alpha$ : angle of incidence at $\mathrm{G}, \varepsilon^{\prime}$ : the angle of refraction at $\mathrm{A}, \alpha^{\prime}$ : angle of refraction at $\mathrm{G}, \mathrm{H}$ : light spot at $\mathrm{H}$ on the detector plane, $l_{1}$ : the distance between the optical window and the reflection point (B) on the electrode, $l_{2}$ : the distance between the optical window and the position sensitive detector (PSD), $l$ : the distance between the electrode and the photodetector, $s:$ the length of the electrode in the solution, $h:$ the distance between the solution level and the reflection point. $y$ is the vertical distance between the light spot on the cantilever and the light spot on the PSD. 


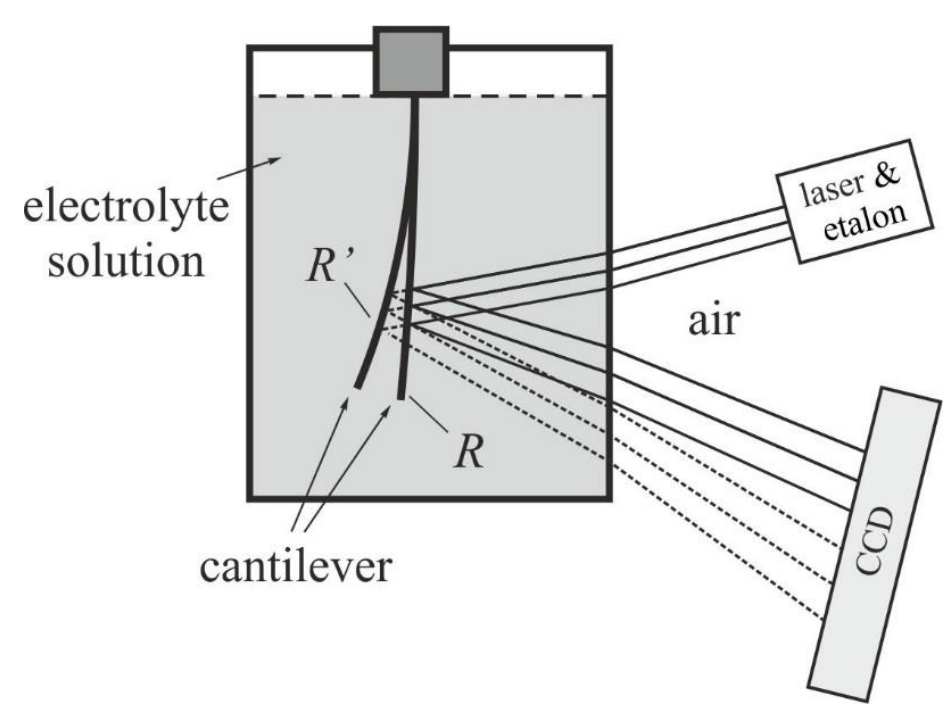

Figure 6: The MOS setup in electrochemical systems.

As it can be seen in Fig. 5, $l$ is the distance between the electrode and the photodetector, $l_{1}$ is the distance between the optical window and the reflection point (B) on the electrode, $l_{2}$ is the distance between the optical window and the detector plane, and $s$ is the length of the electrode in the solution, respectively. The angle of incidence of the light beam coming directly from the laser (in air) is $\varepsilon$. Because of the refraction at A the direction of the beam changes, the new direction of it (in the solution) is $\mathrm{AB}$, the angle of refraction is $\varepsilon^{\prime}$. The laser beam arriving from the direction $\mathrm{AB}$ is reflected at point $\mathrm{B}$ on the surface. The direction of the reflected beam (which strikes the surface of the optical window with an angle of incidence of $\alpha$ ) is BG. Due to the refraction at $\mathrm{G}$, the direction of the reflected beam changes again, the new direction of it (in air) is $\mathrm{GH}$, and the angle of refraction is $\alpha^{\prime}$. The reflected beam results in a light spot at $\mathrm{H}$ on the detector plane. According to the above considerations, if the radius of curvature of the electrode changes, a displacement of the light spot $(\Delta y)$ on the position sensitive detector can be observed.

The vertical distance $y$ between the light spot on the cantilever and the light spot on the PSD can be expressed with the help of the corresponding triangles:

$$
y=l_{1} \tan \alpha+l_{2} \tan \alpha^{\prime}
$$

and by neglecting the thickness of the optical window

$$
l_{1}+l_{2}=l
$$


From Fig. 5 and from Fig. 7 (in which the corresponding segment of the electrode with the incident and reflected light beam is magnified) we can see that

$$
\alpha+\varepsilon^{\prime}=\beta,
$$

and:

$$
\frac{\beta}{2}=\frac{\pi}{2}-\left(\omega-\varepsilon^{\prime}\right)
$$

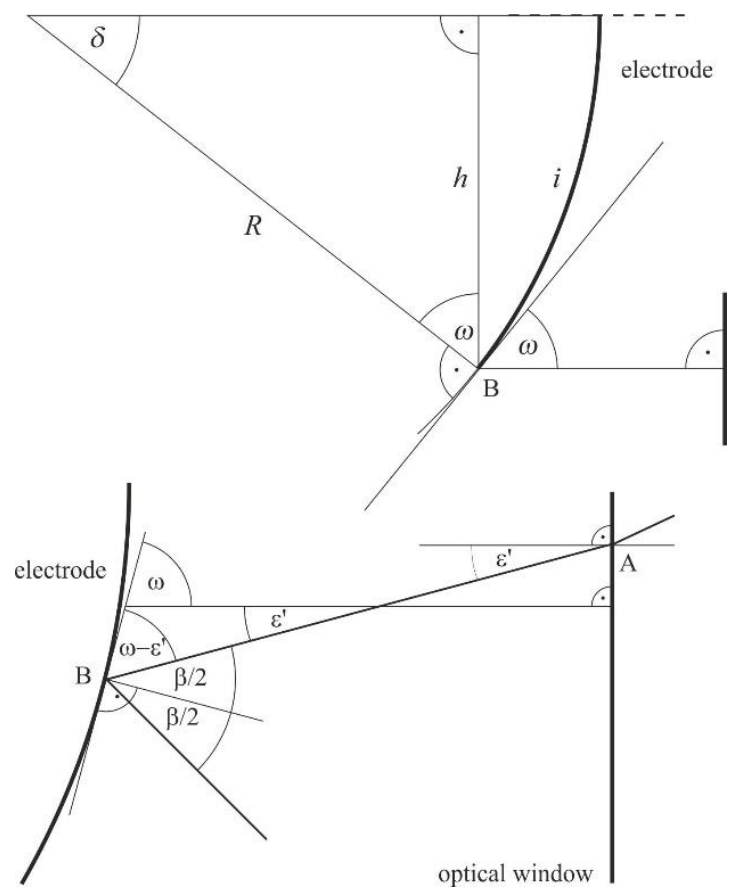

Figure 7: A magnified segment of the electrode with the incident and reflected light beam (see Fig. 5).

Taking into account the rectangle triangle shown in Fig. 7 the angle $\delta$ can be expressed as

$$
\delta=\frac{\pi}{2}-\omega
$$

By combining Eqs. (12)-(14) one obtains

$$
\alpha=2 \delta+\varepsilon^{\prime}
$$

To express $\alpha$, which is the angle between the normal to the optical window and the light beam exiting the electrochemical cell, we can use Schnell's law: 


$$
\frac{\sin \alpha^{\prime}}{\sin \alpha}=n_{\mathrm{s}}
$$

From Eq.(10) we have:

$$
y=l_{1} \tan \alpha+l_{2} \frac{\sin \alpha^{\prime}}{\sqrt{1-\sin ^{2} \alpha^{\prime}}}
$$

and, with Eqs. (15) and (16)

$$
y=l_{1} \tan \left(2 \delta+\varepsilon^{\prime}\right)+l_{2} n_{\mathrm{s}} \frac{\sin \left(2 \delta+\varepsilon^{\prime}\right)}{\sqrt{1-n_{\mathrm{s}}^{2} \sin ^{2}\left(2 \delta+\varepsilon^{\prime}\right)}}
$$

Eq.(18) is suitable (at least in principle) for calculating $y$ using experimentally measurable parameters: the values of $\delta$ and $\varepsilon^{\prime}$ can be determined knowing the incident angle of the light beam, the refractive index and the radius of curvature of the cantilever.

Using Eq. (15) we can derive simpler equations for the change of $y$. Differentiating the function $y(\delta)$ with respect to $\delta$ we have:

$$
\frac{\mathrm{d} y}{\mathrm{~d} \delta}=2 l_{1} \frac{1}{\cos ^{2}\left(2 \delta+\varepsilon^{\prime}\right)}+2 l_{2} n_{\mathrm{s}} \frac{\cos \left(2 \delta+\varepsilon^{\prime}\right)}{\left[1-n_{\mathrm{s}}^{2} \sin ^{2}\left(2 \delta+\varepsilon^{\prime}\right)\right]^{3 / 2}}
$$

By taking into account Eqs.(15) and (16):

$$
\frac{\mathrm{d} y}{\mathrm{~d} \delta}=2 l_{1} \frac{1}{\cos ^{2} \alpha}+2 l_{2} n_{\mathrm{s}} \frac{\cos \alpha}{\cos ^{3} \alpha^{\prime}}
$$

It is clear that $\alpha^{\prime} \geq \alpha$, since the solution is optically denser than air, consequently $\cos ^{3} \alpha^{\prime} \leq \cos \alpha^{\prime} \leq \cos \alpha \leq 1$, and therefore

$$
\frac{\cos \alpha}{\cos ^{3} \alpha^{\prime}} \geq 1
$$

Since $\mathrm{d} \delta \approx i \mathrm{~d}(1 / R)$, by using Eq. (19) the following equation can be obtained:

$$
\frac{\mathrm{d} y}{\mathrm{~d}(1 / R)}=2 i l_{1} \frac{1}{\cos ^{2}\left(\frac{2 i}{R}+\varepsilon^{\prime}\right)}+2 i l_{2} n_{\mathrm{s}} \frac{\cos \left(\frac{2 i}{R}+\varepsilon^{\prime}\right)}{\left[1-n_{\mathrm{s}}^{2} \sin ^{2}\left(\frac{2 i}{R}+\varepsilon^{\prime}\right)\right]^{3 / 2}}
$$

Now we can express $\Delta y$ (that is the change of the position of the light spot on the PSD) by using Schnell's law $\left(\frac{\sin \varepsilon}{\sin \varepsilon^{\prime}}=n_{\mathrm{s}}\right)$ and the following assumption: $\Delta(1 / R)$ is small enough to use first-order approximation for the changes, $i \approx h$, and $2 i / R=2 \delta<<\varepsilon^{\prime}$. 
According to the above considerations:

$$
\Delta y \approx \frac{\mathrm{d} y}{\mathrm{~d}(1 / R)} \Delta(1 / R) \approx\left[2 h l_{1} \frac{1}{1-n_{\mathrm{s}}^{-2} \sin ^{2} \varepsilon}+2 h l_{2} n_{\mathrm{s}} \frac{\left(1-n_{\mathrm{s}}^{-2} \sin ^{2} \varepsilon\right)^{1 / 2}}{\left(1-\sin ^{2} \varepsilon\right)^{3 / 2}}\right] \Delta(1 / R)
$$

In addition, if $l_{1}<<l_{2}$.

$$
\Delta y \approx 2 \operatorname{lh} n_{\mathrm{s}}\left[\frac{\left(1-n_{\mathrm{s}}^{-2} \sin ^{2} \varepsilon\right)^{1 / 2}}{\left(1-\sin ^{2} \varepsilon\right)^{3 / 2}}\right] \Delta(1 / R)
$$

or

$$
\Delta(1 / R) \approx \frac{\Delta y}{2 l h n_{\mathrm{s}}}\left[\frac{\left(1-\sin ^{2} \varepsilon\right)^{3 / 2}}{\left(1-n_{\mathrm{s}}^{-2} \sin ^{2} \varepsilon\right)^{1 / 2}}\right]=\frac{\Delta y}{2 l h n_{\mathrm{s}}} \xi\left(\varepsilon, n_{\mathrm{s}}\right)
$$

The factor $\xi\left(\varepsilon, n_{\mathrm{s}}\right)$ in square brackets in Eq.(25), expressing the effect of the incident angle, is a monotonously decreasing function $\varepsilon$. For $n_{\mathrm{s}}\left(20^{\circ} \mathrm{C}\right) \approx 1.333$ (pure water) and for $\varepsilon=20^{\circ}$ it has the value of $\xi\left(30^{\circ}, 1.333\right)=0.859$, for $\varepsilon=20^{\circ}$ and $n_{\mathrm{s}}\left(20^{\circ} \mathrm{C}\right) \approx 1.420$ (this is the refractive index e.g. of propylene carbonate $\xi\left(20^{\circ}, 1.420\right)=0.855$, respectively. Note that if the deflection of the electrode is small and $\varepsilon$ tends to zero ("normal incidence") we get back the formula derived earlier for perpendicular incident light [116]:

$$
\Delta y \approx 2 \operatorname{lh} n_{\mathrm{s}} \Delta(1 / R)
$$

or

$$
\Delta(1 / R) \approx \frac{\Delta y}{2 l h n_{\mathrm{s}}}
$$

As it can be seen from Eqs. (8), (9), and (27), for the calculation of $\Delta \gamma_{\mathrm{s}}$ only the experimental determination of $\Delta y$ is necessary, if the actual values of $k_{\mathrm{i}}\left(\right.$ or $\left.t_{\mathrm{s}}, E_{\mathrm{s}}, v_{\mathrm{s}}\right), l, h$, and $n_{\mathrm{s}}$ are known.

Unfortunately, in many papers reporting results on electrochemical bending beam experiments with optical detection, schemes of experimental arrangements can be found in which the direction of the reflected beam before and after passing the optical window or the air/solution boundary is indicated incorrectly, since the effect of refraction is ignored [see e.g. in $105,116,117,118]$. It is even more regrettable that the effect of refraction is often neglected also in the calculations. In addition no reference is made to the refractive index of the solution, or 
the value of the refractive index of the solution is not indicated. However, refractive indices of aqueous solutions are about $1.33-1.48$. It is evident from the above equations that the complete neglect of the bending of the laser beam due to refraction at the optical window may cause an error of about 25-32\% in the determination of $\Delta \gamma_{\mathrm{s}}$ in aqueous solutions (because of $n_{\mathrm{s}}$ only!), and the error is more pronounced in the case of liquids of higher refractive index. The error is even greater if the incident angle is different from zero. Other possible sources of errors are discussed in [34].

\subsubsection{Interferometric detection}

The deflection of a strip or a plate can also be measured interferometrically [119]. Fig. 8 shows the principle of the electrochemical Kösters laser interferometer, which can be used for the determination of changes of surface stress by the resulting deformation of an elastic plate. (Not shown in the Figure are the adjustment devices for rotating and turning the optical components in three dimensions, and tilting the electrochemical cell with the quartz plate at its bottom with respect to the prism, and the damping elements.) The Kösters laser interferometer (Kösters-prism [120] interferometer) is a laser-illuminated double-beam interferometer. The main advantage of this type of interferometer is its high immunity to environmental noise due to the close vicinity of the two interfering beams. This immunity makes it an ideal tool for highprecision measurements. The central constituent of the interferometer is the Kösters-prism beam splitter, which produces two parallel coherent beams. The two reflected beams recombine in the prism, and an interference pattern can be observed. Usually, the Kösters prisms are constructed of two halves of fused silica joined together along a coated surface, which acts as a beam splitter. The angles of the prism halves are $30^{\circ}-60^{\circ}-90^{\circ}$, with high angular accuracy, and one long cathetus side is semi-transparent (the reflection and transmission coefficients are equal). The semi-transparent layer performs an equal intensity division of the beam, reflecting half and transmitting half, imparting a 90 degree phase lag in the transmitted beam. This division and phase shift gives the Kösters prism its interferometric properties: the beam reflected from one side of the prism interferes constructively or destructively with the beam transmitted from the other side.

As it can be seen in Fig. 8, the light from the laser is reflected by mirrors M1 and M2 in the direction perpendicular to the entrance side of the prism. The point of entrance determines the distance of the two beams emerging from the base of the prism. They are reflected at a nearly zero angle of incidence from the plate. The interfering light leaves the Kösters prism 
through the exit side, and it is projected (using the mirrors M3 and M4) onto a screen with a hole of a given diameter and a photodiode behind it. The deformation of the plate causes sinusoidal changes of the light intensity at the point of measurement in the interference pattern.

The difference between the optical path lengths $\left(2 \times \Delta Z_{\mathrm{C}}\right)$ can be determined from the change in light intensity detected by the photodiode. The height $\Delta Z_{C}$ of the center of the plate with respect to a plane at a given radius yields $\Delta \gamma_{\mathrm{s}}$ from the appropriate form of Hooke's law

$$
\Delta \gamma_{\mathrm{S}}=k \Delta Z_{\mathrm{C}}
$$

The sensitivity is of the order $0.1 \mathrm{~nm}$ with respect to $\Delta Z_{\mathrm{C}}$ and $1 \mathrm{mN} / \mathrm{m}$ with respect to $\Delta \gamma_{\mathrm{s}}$. The constant $k$ in Eq.(28) is determined by the mechanical properties of the quartz plate (radius $R$ ) and by the type and quality of the support at the edge of the plate.

Choosing a circular AT-cut quartz plate with a thin metal layer on it in contact with the solution being the working electrode in an electrochemical cell provides the advantage to measure simultaneously surface energy, mass and charge [57,60,62-64,121]. (If the metal layers on both sides of the quartz disc are connected to an appropriate oscillator circuit, the device can be used as an electrochemical quartz crystal nanobalance.) In addition, since the light beams do not pass the air/solution interface, the effects of light refraction at the surface are excluded.

Even though there are great advantages of the interferometric detection, there are several problems connected with this method. For instance, the shape and the magnitude of the deformation $Z(r, \varphi)$ as a function of the radial distance $r$ and the angle $\varphi$ depends on the type of support at the edge of the circular plate. The largest deformation and thus the highest sensitivity for measurements of the surface stress change is expected for the "unsupported" plate. A plate is also unsupported if a mounting is present but exerts no forces on the edge. Evidently, the design and realization of such a device is very difficult [57]. In the case of evaporated/sputtered metal layers the high surface stress changes may cause problems with the adhesion of the films. In addition, no absolutely satisfactory solution has been found for the problem of making reliable electrical connections to the metal layers on the quartz crystal. 


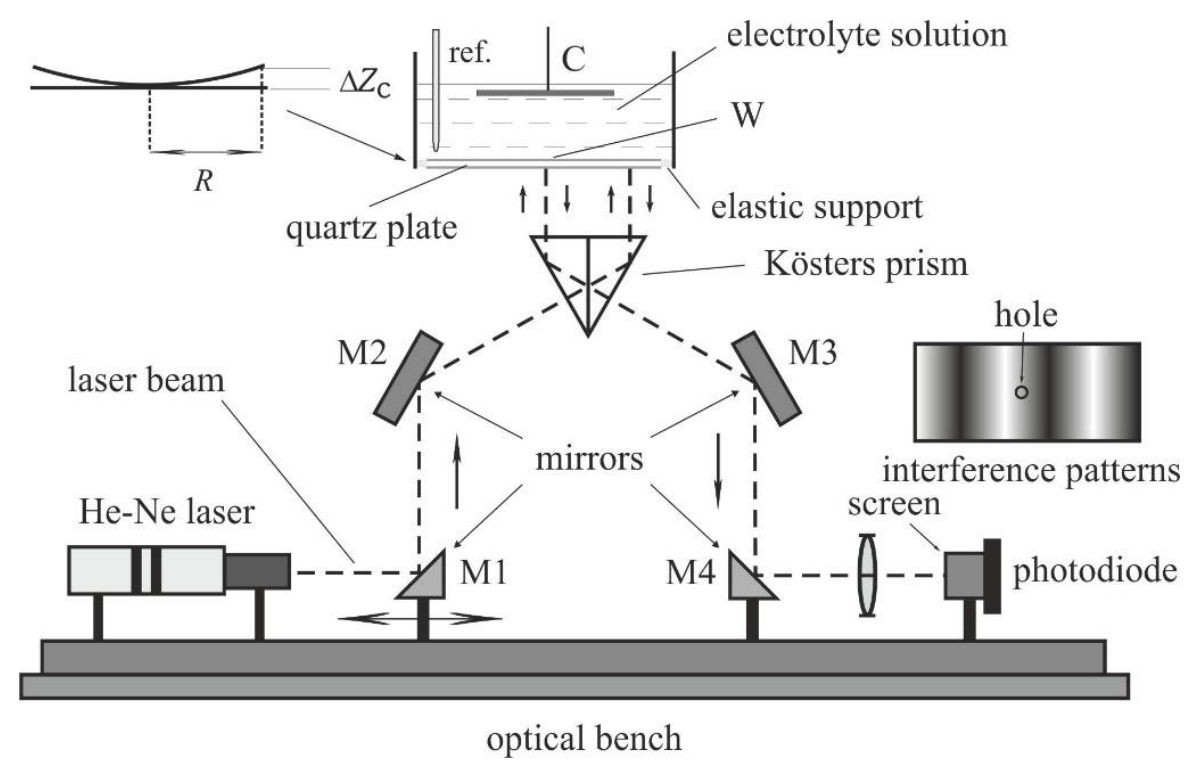

Figure 8: Interferometric setup for electrochemical purposes with $\mathrm{He}-\mathrm{Ne}$ laser and Kösters prism. W: working electrode, C: counter electrode, ref.: reference electrode, M1, M2, M3, M4: mirrors.

\subsubsection{Detection by microscopy}

A quite elegant method to measure the bending of a strip or a plate is to use the scanning tunneling microscope (STM) [58,122-125]. The STM may be used then as a means to simultaneously investigate the structure of the surface (Fig. 9). Unfortunately, even this method is not without pitfalls. In electrolyte solutions there is double layer like structure also around the STM tip. Consequently, there are some interactions between the tip of the STM and the sample that seem to be unavoidable. These are: long range electrostatic interactions between electrical (electrochemical) double layers), and structural/dispersion/hydration forces that dominate the interaction at very short ranges. Most of these contributions have been widely studied but some are marginally understood. The repulsion of two double layers was discussed e.g. in $[126,127,128]$. As it has been noted in [129] “ ... one can lift solids by the electrical forces in the double layer". In experiments reported in [125] a small circular portion of the liquid was removed by a syringe in the vicinity of the tip. According to the authors with this simple procedure the tip remained dry and the electrochemical offset current with its concomitant noise was eliminated.

The values of the surface stress changes derived from the Stoney formula were corrected for the small area not covered by the solution. The uncertainty incurred by this procedure has 
been estimated at most 5\%. Obviously, in this setup the error due to the interaction between double layers is eliminated, but a new source of error, namely that due to the creation of a three phase boundary, is introduced. It is well known, that in a three-phase system there is a greater likelihood of surface contamination from organic and oxygen impurities present in the gas phase [49]. On the other hand, the wetting of such metals as gold and platinum is still a subject of controversy among those who consider these metals to be hydrophobic in nature and others who report low or zero contact angle. It is clear, that if the surface tension of the liquid-gas interface or/and the contact angle changes during the experiment, the results obtained may be incorrect. As pointed out in [37], another source of error, which can be important, arises because the exact elastic behavior of membranes is strongly dependent on the boundary conditions, which are not well defined in many experiments.

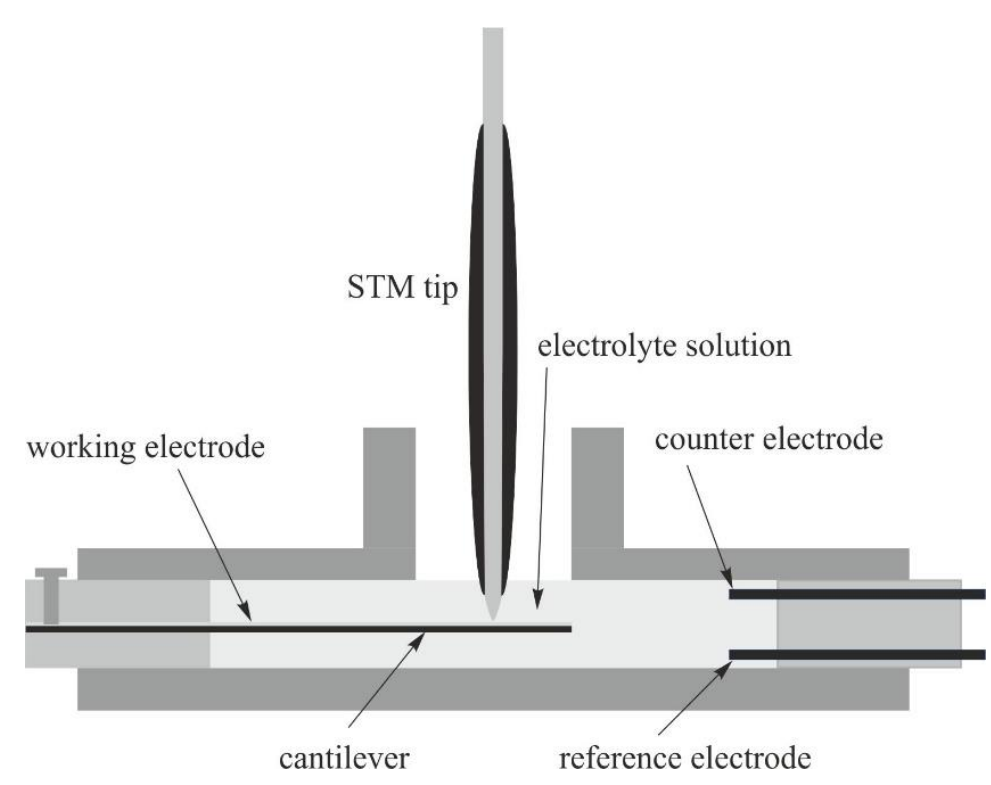

Figure 9: Schematic illustration of a typical arrangement for STM studies at the solid/liquid interface which allows simultaneously to measure the bending of the cantilever when the electrode potential is varied.

\section{Conclusions}

In their famous textbook 'Electrochemical Methods' A.J. Bard and L.R. Faulkner wrote in 1980 that "Examining interfacial structure at a solid surface is extremely difficult ... Measurements of surface tension and surface stress are not easy, but a great deal of attention has been 
paid to them recently; and there is reason for optimism about the future in this area" [130]. In retrospect, this prediction did not come true completely, but over the years very interesting results have been obtained in this field. Especially, the "bending beam" or "bending plate" ("bending cantilever", "laser beam deflection", "wafer curvature", etc.) methods with optical detection (PSD or interferometer) seem to be promising. However, even these methods are not without potential problems. It is necessary to be flexible and to choose the most appropriate method for each particular case. We hope that this short review will help the interested reader to have a glance at the huge progress in this new and developing field.

Funding and acknowledgement: This research has received funding from the Hungarian Scientific Research Fund (OTKA) under Grant Agreement number K 109036. 


\section{References}

1. S. Trasatti, R. Parsons, "Interphases in systems of conducting phases”, Pure \&Appl Chem 58 (3) 437-454 (1986).

2. R. Parsons, “Equilibrium properties of electrified interfaces”, In: J. O’M. Bockris, B. E. Conway (eds) Modern aspects of electrochemistry, Vol. 1. Academic Press, New York (1954).

3. D.C. Grahame, "The electrical double layer and the theory of electrocapillarity", Chem. Rev. 41 (3) 441-501 (1947).

4. P. Delahay "Double layer and electrode kinetics", Interscience, New York (1965).

5. R. Aveyard, D. Haydon, "An introduction to the principles of surface chemistry", Cambridge University Press, Cambridge, (1973).

6. J.O’M. Bockris, B.E. Conway, E. Yeager (eds) “Comprehensive treatise of electrochemistry”, Vol. 1. Plenum Press, New York (1980).

7. H. Girault, D. Schiffrin, "Electrochemistry of liquid-liquid interfaces”, In: A.J. Bard (ed), Electroanalytical chemistry, Vol. 15. Marcel Dekker, New York (1989).

8. G. Lippmann, "Relations entre les phénomènes électriques et capillaries" Ann. Chim. Phys. (5) 494-549 (1875).

9. A.W. Adamson, I. Ling, "The status of contact angle as a thermodynamic property", Adv. Chem. Ser. (43) 57-73 (1964).

10. C. Herring, "Some Theorems on the Free Energies of Crystal Surfaces", Phys. Rev. 82 (1) $87-93$ (1951).

11. R.G. Linford, "The derivation of thermodynamic equations for solid surfaces", Chem. Rev. 78 (2) 81-95 (1978).

12. G Horányi; G G Láng, Double-layer phenomena in electrochemistry: Controversial views on some fundamental notions related to electrified interfacesJ Colloid Interf Sci 296: (1) pp. 1-8. (2006).

13. G. Láng, K.E. Heusler, "Problems related to the specific surface energy of solid electrodes", Journal of Electroanalytical Chemistry 377 (1) 1-7 (1994).

14. K.E. Heusler, G. Láng, "Surface energies of solid electrodes”, Electrochimica Acta 42 (5) 747-756 (1997).

15. R. Guidelli, "Superficial work and surface stress at solid electrodes: a thermodynamic assessment", J. Electroanal. Chem. (453) 69-77 (1998).

16. G. Láng, K.E. Heusler, "Can the internal energy function of solid interfaces be of a non-homogeneous nature?”, J. Electroanal. Chem. 472 (2) 168-173 (1999).

17. R. Guidelli, "A reply to the question of whether the internal energy of solid interfaces can be of a non-homogeneous nature", J. Electroanal. Chem. 472 (2) 174-177 (1999).

18. G. Valincius, "Electrocapillary equations of solid electrodes", J. Electroanal. Chem. 478 (1-2) 40-49 (1999).

19. J. Lipkowski, W. Smickler, D.M. Kolb, R. Parsons, "Comments on the thermodynamics of solid electrodes", J Electroanal. Chem. 452 (2) 193-197 (1998).

20. P.R. Couchman, W.A. Jesser, D. Kuhlmann-Wilsdorf, "On the concepts of surface stress and surface strain", Surf. Sci. 33 (3) 429-436 (1972).

21. P.R. Couchman, W.A. Jesser, "On the thermodynamics of surfaces”, Surf. Sci. 34 (2) 212-224 (1973). 
22. P.R. Couchman, D.W. Everett, W.A. Jesser, "Remarks on the derivation of the Gibbs-Duhem relation for a surface", J. Coll. Interf. Sci. 52 (2) 410-411 (1975).

23. A.I. Rusanov, "On the thermodynamics of deformable solid surfaces", J. Coll. Interf. Sci. 63 (2) 330-345 (1978).

24. A.I. Rusanov, "Advances in thermodynamics of solid surfaces", Pure and Appl. Chem. 61, 1945-1948 (1989).

25. A.I. Rusanov, “Thermodynamics of solid surfaces”, Surf. Sci. Rep. 23 (6-8) 173-247 (1996).

26. P.R. Couchman and D.W. Everett, "A comment on certain parameters and equations in surface thermodynamics", J. Electroanal. Chem. 67 (3) 382-386 (1976).

27. B.M. Grafov, G. Paasch, W. Plieth, A. Bund, "Connection of the generalized Shuttleworth equation for the elastic spherical electrode with the Laplace formula and the Gibbs adsorption equation", Electrochim. Acta 48 (5) 581-587 (2003).

28. V.A. Marichev, "Structure-mechanical approach to surface tension of solids", Surf. Sci. 600 (19) 45274536 (2006).

29. V.A. Marichev, "Plastic deformation and surface tension of solids", Chem. Phys. Lett. 434 (4-6) 218-221 (2007).

30. V.A. Marichev, "Surface tension of solids. Structure-mechanical approach", Prot. Met. 44 (2) 105-119 (2008).

31. E.M. Gutman, On the thermodynamic definition of surface stress, J. Phys.: Condens. Matter 7 L663-L667 (1995).

32. S. Fletcher, "Surface thermodynamics reconsidered. Derivation of the Gokhshtein relations from the Gibbs potential and a new approach to surface stress", J. Solid State Electrochem. 18, 1231-1238, (2014).

33. E.M. Gutman, "Theoretical problems in solid electrocapillarity” J. Solid State Electrochem. 18 (12) $3217-$ 3237 (2014).

34. G.G. Láng, C. Barbero. Laser Techniques for the Study of Electrode Processes, Monographs in Electrochemistry, F. Scholz (Ed.), Springer, Heidelberg, New York, 2012.

35. E.M. Gutman. Thermodynamic aspects of capillarity and electrocapillarity of solid interfaces. J. Solid State Electrochem. 20 (11):2929-2950 (2016).

36. J.J. Bikerman in Topics in Current Chemistry ;77, Springer-Verlag, Berlin, 1978.

37. G.G. Láng, N.S. Sas, S. Vesztergom, "Experimental Determination of Surface Stress Changes in Electrochemical Systems - Possibilities and Pitfalls", Chem. Biochem. Eng. Q. 23 (1) 1-9 (2009).

38. H. Ibach, "The role of surface stress in reconstruction, epitaxial growth and stabilization of mesoscopic structures”, Surf. Sci. Reports 29 (5-6) 195-263 (1997).

39. K. Ueno, M. Seo, "Study of adsorption of iodide ions on gold electrode by a laser- beam deflection method compared with a piezoelectric technique", J. Electrochem. Soc. 146 (4) 1496-1499 (1999).

40. R.A. Fredlein, A. Damjanovic, J.O’M. Bockris, "Differential surface tension measurements at thin solid metal electrodes", Surf. Sci. 25 (2) 261-264 (1971).

41. R.A. Fredlein, J.O’M. Bockris, "An "electrocapillary” study of the gold-perchloric acid solution interface”, Surf. Sci. 46 (2) 641-652 (1974). 
42. S.N. Sahu, J. Scarminio, F. Decker, "A laser beam deflection system for measuring stress variations in thin film electrodes", J. Electrochem. Soc. 137 (4) 1150-1154 (1990).

43. F. Tian, J.H. Pei, D.L. Hedden, G.M. Brown, T. Thundat, "Observation of the surface stress induced in microcantilevers by electrochemical redox processes", Ultramicroscopy 100 (3-4) 217-223 (2004).

44. S. Cattarin, E. Pantano, F. Decker, "Investigation by electrochemical and deflectometric techniques of silicon dissolution and passivation in alkali”, Electrochem. Commun. 1 (10) 483-487 (1999).

45. S. Cattarin, F. Decker, D. Dini, B. Margesin, "In-situ detection of stress in oxide films during Si electrodissolution in acidic fluoride electrolytes", J. Electroanal. Chem. 474(2) 182-187 (1999).

46. R. Raiteri, H.-J. Butt, M. Grattarola, "Changes in surface stress measured with an atomic force microscope", Scanning Microscopy 12, 243-253 (1998).

47. O.E. Kongstein, U. Bertocci, G.R. Stafford, "In situ stress measurements during copper electrodeposition on (111)-textured Au”, J. Electrochem. Soc., 152 (3) C116-C123 (2005).

48. G.R. Stafford, U. Bertocci, "In situ stress and nanogravimetric measurements during underpotential deposition of bismuth on (111)-textured Au”, J. Phys. Chem. B 110 (31) 15493-15498 (2006).

49. I. Morcos, in 'Specialist Periodical Reports Electrochemistry', Vol 6. In: Thirsk HR (ed) The Chemical Society, Burlington House, London, 65-97 (1978).

50. I. Morcos, H. Fischer, "Determination of the potential of zero charge from capillary liquid rise on metal plates" J Electroanal Chem 17 (1-2) 7-11 (1968).

51. I. Morcos, "Electrocapillary phenomena at the stress-annealed pyrolytic graphite electrode" J Phys Chem. 76 (19) 2750-2753 (1972).

52. I. Morcos, "Determination of Surface Tensions of Liquids from Liquid Meniscus Rise on Partially Immersed Plates" J. Chem. Phys. 55 4125-4126 (1971).

53. I. Morcos, "Surface Tension of Stress- Annealed Pyrolytic Graphite" The Journal of Chemical Physics 57 (4),1801-1802,(1972).

54. I. Morcos, "Electrocapillary Studies on Partially Immersed Mercury- Plated Electrode in DMF- Water Solutions" J. Chem. Phys. 56 (8) 3996-4000 (1972).

55. A. Ya. Gokhshtein, in "Surface Tension of Solids and Adsorption", Nauka, Moscow (1976)

56. M. Seo, T. Makino, N. Sato, "Piezoelectric response to surface stress change of platinum electrode", $J$. Electrochem. Soc. 133 (6) 1138-1142 (1986).

57. L. Jaeckel, G.G. Láng, K.E. Heusler, “A new interferometric method to investigate surface energies at solid electrodes“, Electrochim. Acta 39 (8-9) 1031-1038 (1994).

58. H. Ibach, C.E. Bach, M. Giesen, A. Grossmann, "Potential-induced stress in the solid-liquid interface: $\mathrm{Au}(111)$ and $\mathrm{Au}(100)$ in an $\mathrm{HClO}_{4}$ electrolyte“, Surf. Sci. 375 (1) 107-119 (1997).

59. W. Haiss, "Surface stress of clean and adsorbate-covered solids”, Rep. Prog. Phys. 64 (5) 591-648 (2001).

60. G.G. Láng, M. Seo, K.E. Heusler, "Simultaneous oscillations of surface energy, superficial mass and electrode potential in the course of galvanostatic oxidation of formic acid”, J. Solid State Electrochem. 9 (5) 347-353 (2005).

61. G.G. Láng, K. Ueno, M. Ujvári, M. Seo, “Simultaneous Oscillations of Surface Stress and Potential in the Course of Galvanostatic Oxidation of Formic Acid”, J. Phys. Chem. B 104 (13) 2785-2789 (2000). 
62. G. Láng, K.E. Heusler, "Surface energies of an electrode in equilibrated partially miscible electrolytes", J. Chem. Soc., Farad. Trans. 93 (4) 583-589 (1997).

63. G.G. Láng, K.E. Heusler, "Changes of the specific surface energy of gold due to the chemisorption of sulphate", J. Electroanal. Chem. 391 (1-2) 169-179 (1995).

64. G. Láng, K.E. Heusler, "On the interpretation of specific surface energies measured at solid electrodes changes of specific surface-energy of gold in chloride solution", Russ. J. Electrochem. 31 (8) 759-767 (1995).

65. R.E. Malpas, R.A. Fredlein, A.J. Bard, "The Piezoelectric Detection of Electrode Surfaces Processes", J. Electroanal. Chem. 98 171-180 (1979).

66. M. Seo, K. Ueno, "Piezoelectric detection of changes in surface energy of gold electrode in perchlorate solutions containing iodide ions", J. Electrochem Soc. 143 (3) 899-904 (1996).

67. K. Ueno, M. Seo, "Study of adsorption of iodide ions on gold electrode by a laser- beam deflection method compared with a piezoelectric technique", J. Electrochem. Soc. 146 (4) 1496-1499 (1999).

68. T.R. Beck, "'Electrocapillary curves" of solid metals measured by extensometer instrument”, J. Phys. Chem. 73 (2) 466-468 (1969).

69. K.F. Lin, T.R. Beck, "Surface stress curves for gold", J. Electrochem. Soc. 123 (8) 1145 (1976).

70. T.R. Beck, K.F. Lin, “Surface Stress Curves for Platinum”, J. Electrochem. Soc. 126 (2) 252-256 (1979).

71. T.R. Beck, K.W. Beach, in 'Proceedings of the Symposium on electrocatalysis', ed. M.W. Breiter, The Electrochemical Society, Soft-bound Symposium Series, Princeton, N.J., 1974, pp. 357-364.

72. K.F. Lin, "Some Electrocapillary- Type Behaviors of Gold Electrode”, J. Electrochem. Soc. 125 (7) 10771078 (1978).

73. Á. Horváth, R. Schiller, "The effect of mechanical stress on the potential of the $\mathrm{Ag} / \mathrm{Ag}^{+}$electrode", Phys. Chem. Chem. Phys. 3, 2662-2667 (2001).

74. M. Smetanin, D. Kramer, S. Mohanan, U. Herr, J. Weissmüller "Response of the potential of a gold electrode to elastic strain", Phys. Chem. Chem. Phys. 11, 9008-9012 (2009).

75. P.P. Craig, "Direct Observation of Stress-Induced Shifts in Contact Potentials" Phys. Rev. Lett. 22 (14) 700-703 (1969).

76. K. Unal, H.K. Wickramasinghe, "Nanoscale quantitative stress mapping with atomic force microscopy" Appl. Phys. Lett. 90113111 (2007).

77. J. Weissmüller, R.N. Viswanath, D. Kramer, P. Zimmer, R. Würschum, H. Gleiter, "Charge-induced reversible strain" Science 300 312-315 (2003).

78. D. Kramer, R.N. Viswanath, J. Weissmüller, "Surface-Stress Induced Macroscopic Bending of Nanoporous Gold Cantilevers" Nano Lett. 4 793-796 (2004).

79. M.A. Butler, D.S. Ginley, "In-site measurement of strain during electrodeposition" J Electrochem Soc 134 510-511 (1987).

80. M.A. Butler, D.S. Ginley, "New Technique for Measurement of Electrode Strain during Electrochemical Reactions" J Electrochem Soc 135 (1) 45-51 (1988).

81. A.Ya. Gokhshtein, “The estance method”, Elektrokhimiya 6, 979-985 (1970).

82. A.Ya. Gokhshtein, Electrolysis and surface phenomena. "To the bicentenary of Volta's publication on the first direct-current source”, Physics-Uspekhi 43 (7) 725-750 (2000). 
83. K.M. Dickinson, K.E. Hanson, R.A. Fredlein, "Potentials of zero charge and capacitance minima of polycrystalline gold in sodium fluoride solutions", Electrochim. Acta 37 (1) 139-141 (1992).

84. M. Seo, X.C. Jiang, and N. Sato, "Piezoelectric response to surface stress change of gold electrode in sulfate aqueous solutions", J. Electrochem. Soc. 134 (12) 3094-3098 (1987).

85. G.G. Stoney, “The tension of metallic films deposited by electrolysis”, Proc Roy. Soc. London A32 (82) 172-175 (1909).

86. G.G. Láng, in “Electrochemical Dictionary”, Eds. A.J. Bard, G. Inzelt, F. Scholz, Springer, Berlin, 2008.

87. D. Sander, "The correlation between mechanical stress and magnetic anisotropy in ultrathin films" Rep. Prog. Phys. 62 (5) 809-858 (1999).

88. D. Sander, Z. Tian, J. Kirschner "Cantilever measurements of surface stress, surface reconstruction, film stress and magnetoelastic stress of monolayers“, Sensors 8 (7), 4466-4486 (2008).

89. Brenner A, Senderoff S "Calculation of stress in electrodeposits from the curvature of a plated strip" J. Res. Nat. Bur. Stand. 42 105-123 (1949).

90. V. Lehmann, "On the origin of electrochemical oscillations at silicon electrodes", J. Electrochem. Soc. 143 (4) 1313-1318 (1996).

91. Q. Pei, O. Inganas, "Electrochemical applications of the bending beam method. 1. Mass transport and volume changes in polypyrrole during redox”, J. Phys. Chem. 96 (25) 10507-10514 (1992).

92. K. Asaka, K. Oguro, "Bending of polyelectrolyte membrane platinum composites by electric stimuli: Part II. Response kinetics”, J. Electroanal. Chem. 480 (1-2) 186-198 (2000).

93. G.G. Láng, M. Ujvári, T.A. Rokob, G. Inzelt, "The brush model of the polymer films-analysis of the impedance spectra of Au,Pt|poly(o-phenylenediamine) electrodes”, Electrochim. Acta 51 (8-9) 1680-1694 (2006).

94. M Ujvári, M. Takács, S. Vesztergom, F. Bazsó, F. Ujhelyi, G.G. Láng, "Monitoring of the electrochemical degradation of PEDOT films on gold using the bending beam method", Solid State Electrochem 15(11) 2341-2349 (2011).

95. G.G. Láng, M. Ujvári, F. Bazsó, S. Vesztergom, F. Ujhelyi, "In situ monitoring of the electrochemical degradation of polymer films on metals using the bending beam method and impedance spectroscopy", Electrochim. Acta 73 59-69 (2012).

96. M Ujvári, G.G. Láng, S. Vesztergom, K.J. Szekeres, N. Kovács, J. Gubicza, "Structural changes during the overoxidation of electro-chemically deposited poly(3,4-ethylenedioxythiophene) films" J. Electrochem. Sci. Eng. 6(1) 77-89 (2015).

97. G.G. Láng, M. Ujvári, S. Vesztergom, V. Kondratiev, J. Gubicza, K.J. Szekeres “The Electrochemical Degradation of Poly(3,4-ethylenedioxythiophene) Films Electrodeposited from Aqueous Solutions" Z. Phys. Chem. 230 (9) 1281-1302 (2016).

98. M. Ujvári, S. Vesztergom, Cs.B. Pénzes, G.G. Láng "Changes of the interfacial stress with electrode potential in the Ru|0.1 M perchloric acid system” Electrochem. Comm. 28 111-113 (2013).

99. G.G. Láng, M. Ujvári, S. Vesztergom "Electrochemical Reduction of Perchlorate Ions on Ruthenium" Acta Chim. Slov. 61 (2) 233-240 (2014).

100. C. Klein, R. P. Miller, "Strains and stresses in multilayered elastic structures: The case of chemically vapordeposited ZnS/ZnSe laminates”, J. Appl. Phys. 87 (5) 2265-2272 (2000). 
101. C. Klein, "How accurate are Stoney's equation and recent modifications" J. Appl. Phys. 88 (9) 5487-5489 (2000).

102. J.M. Pureza, M.M. Lacerda, A.L. De Oliveira, J.F. Fragalli, R.A. S. Zanon, "Enhancing accuracy to Stoney Equation" Appl. Surf. Sci. 255 6426-6428 (2009).

103. J.M. Pureza, F. Neri, M.M. Lacerda, "Stoney equation limits for samples deformed as a cylindrical surface" Appl. Surf. Sci. 256 4408-4410 (2010).

104. G.C.A.M. Janssen, M.M. Abdalla, F. van Keulen, B R. Pujada, B. van Venrooy "Celebrating the 100th anniversary of the Stoney equation for film stress: Developments from polycrystalline steel strips to single crystal silicon wafers" Thin Solid Films 517 1858-1867 (2009).

105. T.A. Rokob, G.G. Láng, "Remarks on the electrochemical application of optical methods for the determination of stress in electrodes", Electrochim. Acta 51 (1) 93-97 (2005).

106. J.-F. Vanhumbeeck, J. Proost, "Electrochemical processing of ultrathin metallic oxides featuring in-situ monitoring of growth stress transitions", ECS Trans. 2 (6) 281-294 (2007).

107. J.-F. Vanhumbeeck, J. Proost, "On the contribution of electrostriction to charge-induced stresses in anodic oxide films" Electrochim. Acta 53 (21) 6165-6172 (2008).

108. J.A. Floro, E. Chason, "Measuring Ge segregation by real- time stress monitoring during $\mathrm{Si}_{1-\mathrm{x}} \mathrm{Ge}_{\mathrm{x}}$ molecular beam epitaxy" Appl. Phys. Lett. 69 (25) 3830-3832 (1996).

109. S. Hearne, E. Chason, J. Han, J. A. Floro, J. Figiel, J. Hunter, H. Amano, I. S. T. Tsong "Stress evolution during metalorganic chemical vapor deposition of GaN" Appl. Phys. Lett. 74 356-358 (1999).

110. J. Proost, F. E. Spaepen, "Evolution of the growth stress, stiffness, and microstructure of alumina thin films during vapor deposition”, J. Appl. Phys. 91 (1) 204-216 (2002).

111. Q. Van Overmeere, J.-F. Vanhumbeeck, J. Proost "On the use of a multiple beam optical sensor for in situ curvature monitoring in liquids" Rev. Sci. Instrum. 81045106 (2010).

112. J.-F. Vanhumbeeck, J. Proost "In-situ monitoring of the growth stress evolution during galvanostatic anodizing of aluminum thin films". In:Moffat TP, Stickney J, Deligianni H, Dukovic J (eds) ECS Transactions. Electrochemical Society, Pennington NJ (2007).

113. J.-F. Vanhumbeeck, J. Proost J "On the contribution of electrostriction to charge-induced stresses in anodic oxide films" Electrochim. Acta 53 6165-6172 (2008).

114 Q. Van Overmeere, B. Nysten, J. Proost "In situ detection of porosity initiation during aluminum thin film anodizing" Appl. Phys. Lett. 94074103 (2009)

115. Q. Van Overmeere, F. Blaffart, J. Proost “What controls the pore spacing in porous anodic oxides?" Electrochem. Comm. 12 1174-1176 (2010).

116. G.G. Láng, M. Seo, "On the electrochemical applications of the bending beam method" J. Electroanal. Chem. 490 (1-2) 98-101 (2000).

117. G.G. Láng, T.A. Rokob, G. Horányi, "Comments on "Observation of the surface stress induced in microcantilevers by electrochemical redox processes" Ultramicroscopy 104 (3-4) 330-332 (2005).

118. G.G. Láng, Comment on "In Situ Measurements of Stress-Potential Coupling in Lithiated Silicon" [J. Electrochem. Soc., 157, A1253 (2010)], "On Plastic Deformation and Fracture in Si Films during Electrochemical Lithiation/Delithiation Cycling" [J. Electrochem. Soc., 160, A1885 (2013)], and "Real-Time Stress 
Measurements in Germanium Thin Film Electrodes during Electrochemical Lithiation/Delithiation $\mathrm{Cy}$ cling" [J. Electrochem. Soc., 162, A2840 (2015)], J. Electrochem. Soc. 163 (7) Y15-Y16. (2016)

119. G.G. Láng, Application of the Koesters Interferometer in Electrochemistry In: D. Halsey, W. Raynor (eds.) Handbook of Interferometers: Research, Technology and Applications. Hauppauge: Nova Science Publishers, 591-604 (2009).

120. W. Kösters, Interferenzdoppelprisma für Messzwecke, German patent: 595211, 1934

121. K.E. Heusler, G. Láng, „On the Interpretation of Specific Surface Energies at Solid Electrodes. Changes of the Surface Energy of Gold in Chloride Solution”, Elektrokhimiya 31 826-835 (1995).

122. W. Haiss, J.K. Sass, "Adsorbate-induced surface stress at the solid electrolyte interface measured with an STM", J. Electroanal. Chem. 386 (1-2) 267-270 (1995).

123. W. Haiss, J.K. Sass, "Apparent thermodynamic inconsistencies in surface stress measurements of copper underpotential deposition on Au( 111) electrodes", J. Electroanal. Chem. 410 (1) 119-124 (1996).

124. W. Haiss, J.K. Sass, "Quantitative surface stress measurements on $\mathrm{Au}(111)$ electrodes by scanning tunneling microscopy", Langmuir 12 (18) 4311-4313 (1996).

125. W. Haiss, R.J. Nichols, J.K. Sass, K.P. Charle, "Linear correlation between surface stress and surface charge in anion adsorption on $\mathrm{Au}(111)$ ", J. Electroanal. Chem. 452 (2) (1998) 199-202.

126. J. O'M. Bockris, R. Parry-Jones, "Determination of the Relative Electrode Potential of an Uncharged Metal in Solution", Nature 171, 930-931 (1953).

127. J. O'M. Bockris, S.D. Argade, "Dependence of friction at wet contacts upon interfacial potential", J. Chem. Phys. 50 (10) 1622-1623 (1969).

128. J. O'M. Bockris, R.K. Sen, "Variation of the coefficient of friction with potential for a solid-solution contact: A revised calculation", Surf. Sci. 30 (1) (1972) 237-241.

129. J. O’M. Bockris in 'Electrochemistry in Transition', Eds. O.J. Murphy, S. Srinivasan, B.E. Conway, Plenum Press, New York, 1992, p. 288.

130. A.J. Bard, L.R. Faulkner, Electrochemical Methods, John Wiley\&Sons, New York, 1980, pp. 499-500. 